\title{
Anzeige neuer Zeitschriften
}

Journal of the History of Biology. Editor: Everett Mendelsohn, Harvard University. Dem Advisory Board gehören u.a. an: Georges Ganguilhem (Frankreich), K. E. Rothschun (Deutschland), Joseph Needham (England). 2 Hefte jährlich. \$ 8.50. Bestellung: Harvard University Press, 79 Garden Street, Cambridge (Mass. 02138, u S A).

Das erste, schön gedruckte Heft enthält eine Reihe wertvoller Aufsätze aus den Gebieten der Biologiegeschichte. Es ist sehr erfreulich, daß die so reiche Geschichte der Biologie, die in den medizinhistorischen Zeitschriften oft zu kurz kommt, ein eigenes Organ erhalten hat, wobei Themen aus der neueren Biologiegeschichte (Claude Bernard, August Weismann, Sherrington, Concept of integrative action), T.H. Morgan (Efficacy of natural selection) im Vordergrund stehen. Es ist zu hoffen, daß diese Zeitschrift berufen ist, eine Lücke im biologiehistorischen Schrifttum auszufüllen und zu einem Kristallisationspunkt der Biologiegeschichte zu werden.

Journal of the Society for the Bibliography of Natural History.

Die von der Society for the Bibliography of Natural History, c/o British Museum (Natural History), Cromwell Road, London S. W. 7 herausgegebene, im 5. Jahrgang stehende, in unregelmäßiger Folge erscheinende Zeitschrift befaßt sich mit der Bibliographie der Naturgeschichte im weitesten Sinn. Unter den Originalartikeln von Vol. 5, Part 1 (September 1968), dürfte der Aufsatz von G. D. R. Bridson, The Zoological Record-a centenary appraisal, allgemeineres Interesse verdienen, ebenso der Artikel von Clifford M.Nelson (englisch) über die Geschichte von der Herkunft und Natur der Ammoniten (Agricola, C. Geßner u.a)und derjenige von D. R. Bowley und H.M. Sмiтh über den Nomenclator Zoolo. gicus von Louis Agassiz. Buchbesprechungen bereichern den Inhalt. Im weiteren gibt die genannte Gesellschaft Faksimileausgaben (als erste Alexander von Humboldts Essai sur la Géographie des Plantes [1807]) heraus.

Die erfreuliche Tatsache eines vermehrten Interesses für die Biologiegeschichte geht auch aus folgender Mitteilung hervor.

Als Cercle d'étude historique des sciences de la vie vereinigte sich 1965 in Paris eine Gruppe historisch interessierter Biologen, deren Ziel es ist, sich in gemeinsamer Arbeit gegenseitig anzuregen und größere Themenkreise untereinander aufgeteilt zu studieren. Sie gab zu Anfang dieses Jahres als erstes Heft einer Reihe Histoire et Biologie mehrere Aufsätze heraus, die Vesals vivisektorische Tätigkeit zum Gegenstand haben. Anfragen sind zu richten an: Cercle d'étude historique des sciences de la vie, Laboratoire d'Ethnobotanique, Muséum National d'Histoire Naturelle, rue Cuvier 57, Paris $V^{\mathrm{e}}$. 